\title{
AVALIAÇÃO DA IMPEDÂNCIA DE TRANSFERÊNCIA DE CABOS COAXIAIS RG213 E RGC213 DE FABRICANTES DISTINTOS
}

\author{
Caio Luminatti Andrade ${ }^{1}$, Valeria L. da Silva ${ }^{1}$, Marcela Silva Novo ${ }^{2}$ e Kenedy Marconi \\ Geraldo Santos ${ }^{2,3}$ \\ ${ }^{1}$ SENAI CIMATEC, ${ }^{2}$ Universidade Federal da Bahia-UFBA, ${ }^{3}$ Instituto Federal da Bahia- \\ IFBA \\ E-mails: luminatticaio@yahoo.com.br, valeria.dasilva@fieb.org.br, msnovo@gmail.com e \\ kenedymarconi@gmail.com
}

\section{RESUMO}

Atualmente vivemos numa era de contínuos avanços tecnológicos, tanto em nosso cotidiano quanto na indústria. Isto implica naturalmente na busca por melhorias na qualidade de processos e produtos. Cabos coaxiais são de particular importância neste contexto devido à sua utilização em sistemas automotivos, telecomunicações, radiofusão, áudio, vídeo dentre outros. Sobre a qualidade da blindagem de cabos coaxiais, esta deveria evitar a propagação de ondas eletromagnéticas (OEM) do interior do cabo para o meio externo e vice versa. Este fenômeno pode ocorrer caso sejam utilizados materiais de baixo rendimento na sua concepção, o que acontece quando fabricantes buscam reduzir custos de produção. Uma forma de avaliar a qualidade desta blindagem frente a propagação de OEM, é através da caracterização da sua impedância de transferência que pode ser obtida por métodos normatizados, como o método triaxial, conforme apresentado em algumas Normas internacionais como, por exemplo, a IEC 62153-4. Sob estas considerações este artigo apresenta, resultados da impedância de transferência de cabos coaxiais do tipo RG213 de diversos fabricantes, na faixa frequência de $10 \mathrm{kHz}$ até $30 \mathrm{MHz}$, determinada experimentalmente através da utilização de um aparato confeccionado e baseado no método triaxial.

\section{INTRODUÇÃO}

Frente aos avanços tecnológicos que tem ocorrido com o passar dos anos nas mais variadas áreas de conhecimento, em especial o segmento automotivo tem apresentado um grande crescimento no que se refere a eletrônica embarcada. A cada ano que passa, o emprego de equipamentos elétricos e eletrônicos em um veículo automotor aumenta consideravelmente, onde são aplicadas soluções automatizadas de conforto, segurança, sinalização dentre outras essenciais onde centrais e módulos eletrônicos se comunicam através de redes para tomada das mais variadas decisões na gestão embarcada do automóvel.

Assim como na indústria automotiva, com essa crescente demanda pela utilização de sistemas eletroeletrônicos, tanto em nosso cotidiano quanto em indústrias assim como em áreas de aplicação de produtos eletromédicos, no segmento de telecomunicações, informática dentre outros, torna-se cada vez mais evidente a necessidade de obtenção de compatibilidade 
eletromagnética entre essa grande quantidade de dispositivos a fim de assegurar o funcionamento destes dispositivos que muitas vezes seu funcionamento indevido pode ocasionar riscos a saúde humana e até mesmo resultar em prejuízos financeiros para grandes indústrias.

Muitos destes equipamentos eletroeletrônicos operam em conjunto com diversos outros sistemas e necessitam muitas vezes de meios para se comunicarem como exemplo de redes de comunicação utilizadas para envio, recebimento e compartilhamento de informações. Dentre alguns dos meios de comunicação utilizados para tal, destacam-se os cabos coaxiais que são utilizados fortemente em diversas áreas, como na área automotiva em sistemas de transmissão de radiofusão, circuitos fechados de TV, antenas UHF/VHF, informática, sistema de TV a cabo, ligações de câmeras, automação dentre outras aplicações como para transmissão de sinais de radiofrequência entre equipamentos em laboratórios de ensaio e calibração.

Sobre a qualidade de cabos coaxiais no que diz respeito a sua eficiência de blindagem, não deveria haver transmissão de sinais e ou perturbações do ambiente externo para o interior do cabo assim como os sinais no seu interior não deveriam ser transmitidos para o exterior do cabo. Assim, a eficiência de blindagem de cabos coaxiais é um parâmetro importante para garantir a compatibilidade eletromagnética de sistemas eletroeletrônicos que são conectados por estes meios de comunicação [1]. Seu desempenho pode ser seriamente afetado se forem utilizados materiais de baixa qualidade na sua construção, o que muitas vezes acontece devido a estratégias tomadas por fabricantes com o objetivo de redução de custos de produção.

Uma das formas de determinar sobre a qualidade da blindagem de cabos coaxiais contra perturbações eletromagnéticas se dá pela caracterização da impedância de transferência $\left(Z_{T}\right)$ e eficiência de blindagem (EB). A impedância de transferência relaciona a queda de tensão existente na blindagem de um cabo coaxial de comprimento definido com a corrente elétrica que flui por este circuito [2], [3] e [4]. Cabos mais suscetíveis a perturbações eletromagnéticas apresentarão maiores valores de impedância de transferência do que cabos mais robustos em termos de qualidade da blindagem.

A série de normas IEC 62153-4 apresentam métodos de teste para determinação da qualidade da blindagem de cabos. Dentre os métodos utilizados para determinação da $Z_{T}$ está o triaxial, o de injeção de linha, câmara reverberante, o de acoplamento por sondas de corrente dentre outros [3]. Neste artigo é utilizado o método triaxial para obtenção da $Z_{T}$, que foi escolhido em função da infraestrutura, equipamentos e materiais disponíveis para sua aplicação. Neste método podem ser utilizados geradores de radiofrequência (RF), analisadores de rede e receptores super-heteródinos [2], [4].

Mediante estas considerações este estudo apresenta a impedância de transferência obtida experimentalmente de cabos coaxiais do tipo RG213 de diversos fabricantes e tipos diferentes, na faixa frequência de $10 \mathrm{kHz}$ até $30 \mathrm{MHz}$.

As próximas seções deste artigo estarão organizadas da seguinte maneira: nas seções 1 e 2 , será abordada a fundamentação teórica relacionada a $Z_{T}$ e método triaxial. A seção 3 descreverá o arranjo utilizado na determinação da impedância de transferência. A seção 4 apresentará os resultados obtidos a partir dos experimentos e algumas discussões. Por fim, na conclusão estarão as considerações finais. 


\section{IMPEDÂNCIA DE TRANSFERÊNCIA}

A $Z_{T}$ (1) de um cabo coaxial fornece informações sobre o desempenho da blindagem deste cabo. Considerando uma amostra de cabo coaxial com uma de suas extremidades curtocircuitadas, A $Z_{T}$ é a relação entre a queda de tensão $\left(V_{S}\right)$ formada ao longo da blindagem dessa amostra de comprimento $L$ conhecido e a corrente $\left(I_{S}\right)$ que se flui na superfície dessa blindagem [2].

$$
Z_{T}=\frac{V_{S}}{L \cdot I_{S}}
$$

A imunidade de um cabo coaxial, ou seja, a sua eficiência em: evitar que perturbações externas do ambiente adentrem a sua blindagem e se acoplem no seu condutor central causando interferências no sinal propagado; e também a sua eficácia em impedir ou minimizar que o sinal propagado restrito entre o condutor central e a blindagem seja propagado por irradiação para o ambiente; será maior quando o valor da impedância de transferência do cabo for menor, assim são inversamente proporcionais. Uma vez satisfeito um determinado nível de imunidade onde o sinal propagado no interior do cabo não sofra com perturbações externas e o ambiente não receba o que está trafegando no interior do cabo, entende-se que a qualidade deste cabo em relação ao seu desempenho em blindar ondas eletromagnéticas é eficiente.

\section{MÉTODO TRIAXIAL}

O método triaxial [2] consiste em utilizar um determinado arranjo constituído de conectores, tubo metálico e demais elementos para determinar a impedância de transferência de um cabo coaxial. Essa impedância de transferência superficial pode ser então caracterizada através de um equacionamento e obtenção através de medições da: tensão induzida em um circuito secundário do sistema que contempla o cabo sob teste (CST) e os componentes do aparato; e da corrente que circula por esta blindagem de um cabo com comprimento $L$ conhecido.

A Figura 1 apresenta uma representação do aparato utilizado para caracterização da $Z_{T}$.

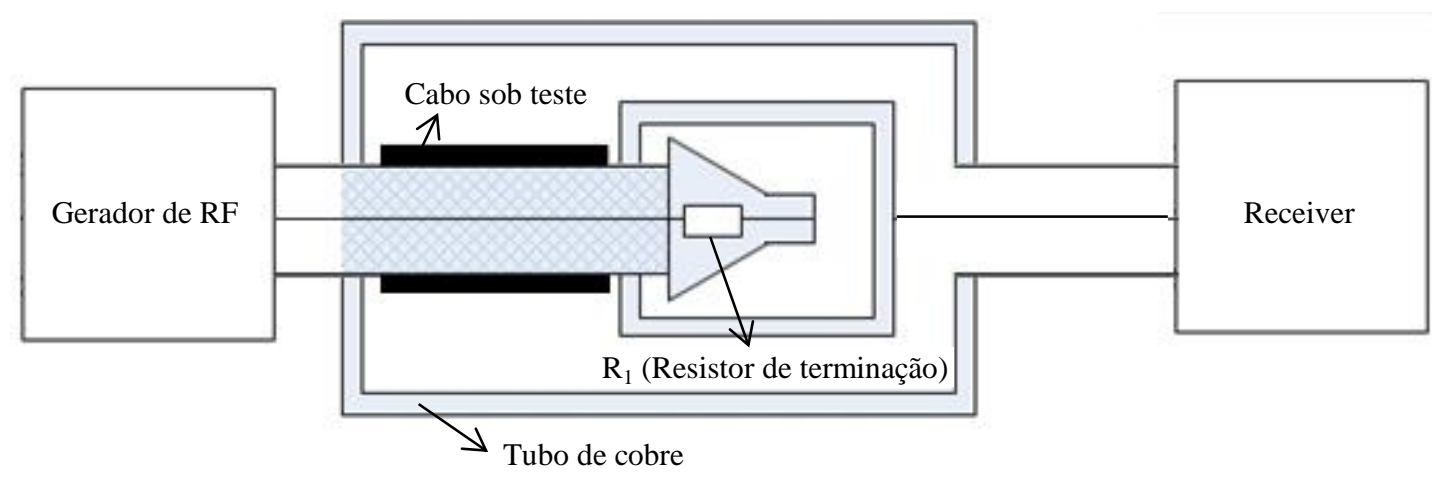

Figura 1. Aparato utilizado baseado no método triaxial.

Dentre as partes utilizadas para elaboração do aparato de testes, o tubo metálico é a um elemento de fundamental importância no sistema visto que forma uma linha de transmissão 
coaxial juntamente com a blindagem do CST e canaliza a corrente injetada no circuito que é requerida para se caracterizar a $Z_{T}[5]$.

A utilização de um aparato que acomode o cabo sob teste horizontalmente possibilita baixa repetibilidade na montagem devido as curvaturas existentes [7]. Porém a utilização de um dielétrico adequado entre o cabo e o tubo minimiza estes efeitos indesejados.

A resistência ôhmica do condutor central do cabo sob teste (CST) que é da ordem de alguns mili ohms pode ser negligenciada na análise devido ao seu valor pequeno valor em comparação ao resistor de terminação $R_{1}$ [8]. Esta é uma estratégia e premissa considerada no sistema para permitir que a medição da tensão sobre a blindagem de comprimento $L$ seja viabilizada. Esta alternativa da forma que é tratada representa uma situação ideal onde o condutor central não tem perdas. Isto é assim tratado porque se considera que este condutor central possui uma resistência à passagem de elétrons muitas vezes menor do que a resistência existente na blindagem do CST.

O método triaxial permite a utilização tanto de um analisador de redes vetorial quanto de um gerador de RF em conjunto com um receiver. Além destes equipamentos ainda poderia ser utilizado um reflectómetro.

O arranjo para aplicação do método triaxial basicamente é composto de duas partes principais, que podem ser representadas por duas linhas de transmissão: uma é denominada como circuito interno ou linha 1 e consiste do CST, formando uma linha de transmissão através da blindagem do CST (considerada como condutor externo da linha) e o condutor central do CST (o condutor interno da linha); a outra linha de transmissão, nomeada como circuito externo ou linha 2, é composta pelo tubo de cobre (condutor externo da linha) e a malha de blindagem do CST (caracterizando a parte interna da linha de transmissão).

No método triaxial, conforme apresentado pelas principais referências [2], [3] e [4], são comumente empregadas 3 tipos de configurações do sistema que envolve as linhas de transmissão e os equipamentos requeridos para a caracterização da $Z_{T}$. Em [5] estas configurações denominadas como procedimentos são também apresentadas. Assim, dentre estas variações do sistema estão:

a) Procedimento 1: com casamento de impedância e com utilização de resistor de damping;

b)Procedimento 2: com carga no circuito interno e sem utilização de resistor de damping;

c) Procedimento 3: sem carga no circuito interno e sem utilização de resistor de damping.

O Procedimento 3 possibilita medições de valores muito baixos, menores do que $1 \mu \Omega / \mathrm{m}$, além do que pode-se desprezar efeitos capacitivos devido a utilização das conexões de curto circuito nas linhas 1 e 2 [6].

Este método triaxial em geral é factível até a frequência de $30 \mathrm{MHz}$ para uma amostra de 1 metro de comprimento e até $100 \mathrm{MHz}$ para uma amostra de 0,3 metros de comprimento, que corresponde a um comprimento elétrico menor do que 1/6 do comprimento de onda da amostra. Assim, evitamos erros nas medições devido aos fenômenos de propagação de maior intensidade que podem ocorrer quando temos a frequência aplicada próximo de $\lambda / 2$ ou $\lambda / 4$ ( $\lambda$ é definido como comprimento de onda), conforme apresentado por [1]. 
Para se determinar a frequência de corte do sistema, próxima de $30 \mathrm{MHz}$ para uma amostra de cabo de 1 metro de comprimento, utiliza-se a equação (2), obtida de [4].

$$
f_{\text {máx }}=\frac{c}{6 \cdot L \cdot \sqrt{\varepsilon_{r}}}
$$

onde,

$f_{\text {máx }}$ é a frequência máxima ou frequência de corte do sistema;

$c$ é a velocidade da luz $\approx 3.10^{8} \mathrm{~m} / \mathrm{s}$;

$L$ é o comprimento da amostra de $\approx 1$ metro;

$\varepsilon_{\mathrm{r}}$ é a permissividade relativa do dielétrico do CST, podendo ser aproximada para o valor de $\sqrt{2}$, de acordo com [1].

Uma técnica que possibilita obter largura de banda mais plana em determinadas medições é a utilização de um resistor de damping [9].

\section{ARRANJO UTILIZADO E INSTRUMENTAÇÃO}

A Figura 2 apresenta o sistema de medição e o aparato utilizado para se determinar a atenuação $a_{\text {meas }}$. Este aparato foi construído no Laboratório de Dispositivos Eletromagnéticos do SENAI - CIMATEC, em Salvador, BA. Até se chegar a esta configuração, foram experimentados alguns modelos anteriores de protótipo. Como o detalhamento do aparato construído não é o foco deste trabalho, uma descrição em detalhes deste artefato será publicada posteriormente em trabalhos futuros.

Dentre os equipamentos utilizados para gerar e medir os sinais desejados está um gerador de sinais de radiofrequência marca Rohde \& Schwarz, modelo SMBV100A. A fim de possibilitar a medição das perdas sobre a blindagem das amostras de cabo avaliadas, o equipamento foi configurado para gerar uma onda senoidal CW (Continuous Wave), com nível de $0 \mathrm{dBm}$. Assim o gerador foi acoplado ao aparato conforme o procedimento adotado. Já a medição da atenuação foi realizada com a utilização de um equipamento denominado por receiver, da marca Rohde \& Schwarz modelo ESCI. Para possibilitar uma medição automatizada integrando os equipamentos utilizados através de interface GBIB (General Purpose Interface Bus), foi utilizado o software EMC32 fornecido pela Rohde \& Schwarz. Através do software foi possível realizar a normalização dos cabos usados para conectar os equipamentos ao aparato, o que possibilitou conhecer sobre as perdas destes cabos para desconsideradas no cálculo da impedância de transferência. 


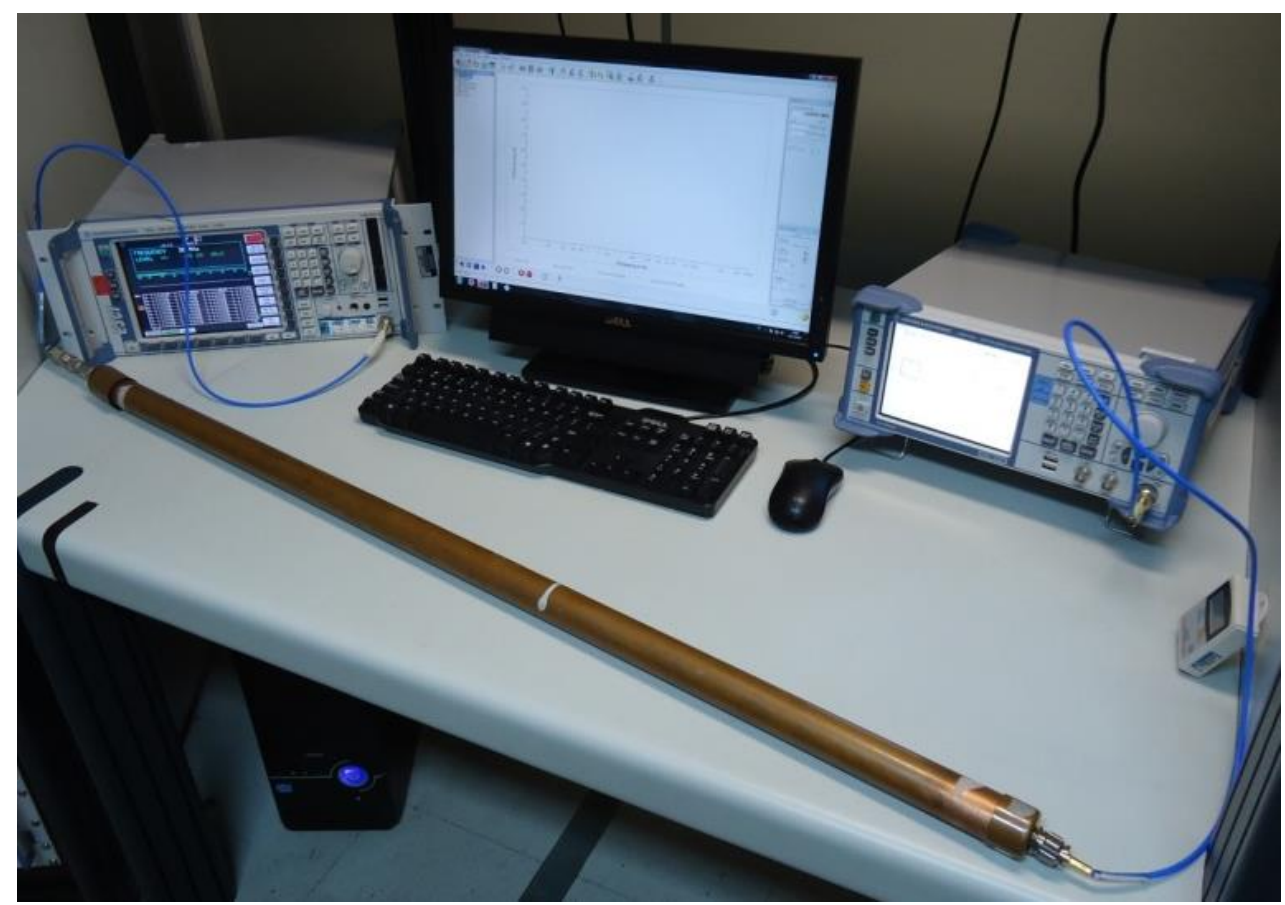

Figura 2. Aparato de testes e sistema de medição.

Para caracterização da $Z_{T}$ das amostras de cabos coaxiais do tipo RG213, foi utilizado o Procedimento 2 apresentado na seção anterior. A equação (3) apresentada por [2] é utilizada para determinação da impedância de transferência para a configuração adotada no Procedimento 2.

$$
Z_{T}=\frac{R_{1}+Z_{0}}{2 \cdot L_{C}} 10^{-\left\{\frac{a_{m e a s}-a_{c a l}}{20}\right\}}
$$

onde,

$Z_{0}$ é a impedância do sistema;

$Z_{l}$ é a impedância característica do circuito interno;

$Z_{T}$ é a impedância de transferência;

$a_{\text {meas }}$ é a atenuação medida;

$a_{c a l}$ é a atenuação dos cabos do sistema de medição. Se um procedimento de normalização de cabos for utilizado deve ser desconsiderada;

$L_{C}$ é o comprimento da blindagem avaliada.

$R_{1}$ é o resistor de terminação do circuito interno;

A atenuação medida $a_{\text {meas }}$, pode ser obtida través de (4).

$$
a_{\text {meas }}=10 \cdot \log _{10}\left(\frac{P_{1}}{P_{2}}\right)
$$

onde, 
$P_{1}$ é o sinal gerado pelo gerador;

$P_{2}$ é o sinal medido pelo receiver.

Em relação ao aparato de testes, a linha 1 foi terminada em uma resistência de $50 \Omega\left(R_{l}\right)$ em função da impedância do gerador e do cabo sob teste serem ambas $50 \Omega$.

\section{MEDIÇÕES E RESULTADOS}

Foram obtidos resultados para a impedância de transferência de cabos coaxiais RG213/U e RGC213, conforme apresentado nas figuras 3 e 4. Os cabos RG213/U, cabos flexíveis, de dois fabricantes distintos, apresentaram impedância de transferência semelhante entre si e muito próximos de algumas referências teóricas apresentadas por [10]. Comparativamente com a curva apresentada por "Kley" a diferença entre as $Z_{T}$ foi menor do que aproximadamente $1 \mathrm{~m} \Omega / \mathrm{m}$ abaixo de $1 \mathrm{MHz}$.

Além disso, pode-se observar que ambos a impedância de transferência obtida experimentalmente e os dados teóricos apresentados por [10], aumentam monotonicamente com a frequência. A partir de aproximadamente $30 \mathrm{MHz}$ ocorre uma divergência que possivelmente se dá pela limitação do método acima desta frequência devido as ressonâncias que ocorrem devido ao comprimento do aparato de medição.



Figura 3. Valores medidos para dois cabos RG213/U versus valores teóricos apresentados em [10].

Já com relação as medições para os cabos semi flexíveis do tipo RGC213 realizadas para 4 fabricantes distintos, os resultados foram divergentes entre si. Pode-se observar que na faixa de frequência de $30 \mathrm{kHz}$ até aproximadamente $100 \mathrm{kHz}$, a resposta é plana apesar da diferença entre a impedância de transferência apresentar grande variação. A partir de $200 \mathrm{kHz}$ observa-se que as amostras "D", "I" e "H" apresentam comportamento levemente semelhante apesar da diferença de $Z_{T}$. Já a amostra " $G$ " se distancia da maioria apresentando a partir de $200 \mathrm{kHz} Z_{T}$ muito menor. 
Esta variação entre os resultados indica uma diferença significativa entre os fabricantes. Isto não é surpreendente já que foram observadas algumas diferenças nas amostras sob teste de cada fabricante. Uma segunda causa que ainda precisa ser investigada é com relação ao posicionamento dos cabos sob teste dentro do aparato de medição. Possivelmente os cabos não ficam dispostos paralelamente ao eixo longitudinal do tubo o que poderia ocasionar erros nas medições.

Essa divergência entre os resultados obtidos para os cabos RGC213 sugere a necessidade de aprofundamento no assunto de forma a investigar a real causa das variações, se de fato são causadas pela diferença da qualidade da blindagem dos diferentes fornecedores ou se são fruto de limitações do aparato utilizado para o estudo.



Figura 4. Analise da $Z_{T}$ no domínio da frequência para cinco fabricantes distintos de cabo RGC213.

Se comparados os dois tipos de cabos, o RG213/U e o RGC213 pode-se notar a divergência entre suas respostas em termos de impedância de transferência. O RG213/U por apresentar melhor flexibilidade mecânica em relação ao RGC213, permite uma melhor acomodação no interior do aparato de testes possibilitando melhor paralelismo na sua disposição em relação ao eixo longitudinal do aparato. Nota-se que a partir de $400 \mathrm{kHz}$ a impedância de transferência dos cabos RGC213 apresenta menor valor em relação aos cabos RG213/U. Isto possivelmente ocorre pela construção dos cabos. O RGC213 por apresentar dupla blindagem sendo a mais interior constituída de blindagem em lâmina, permite uma melhor blindagem para altas frequências por não apresentar frestas na blindagem que possibilitariam transmissão de ondas eletromagnéticas. 


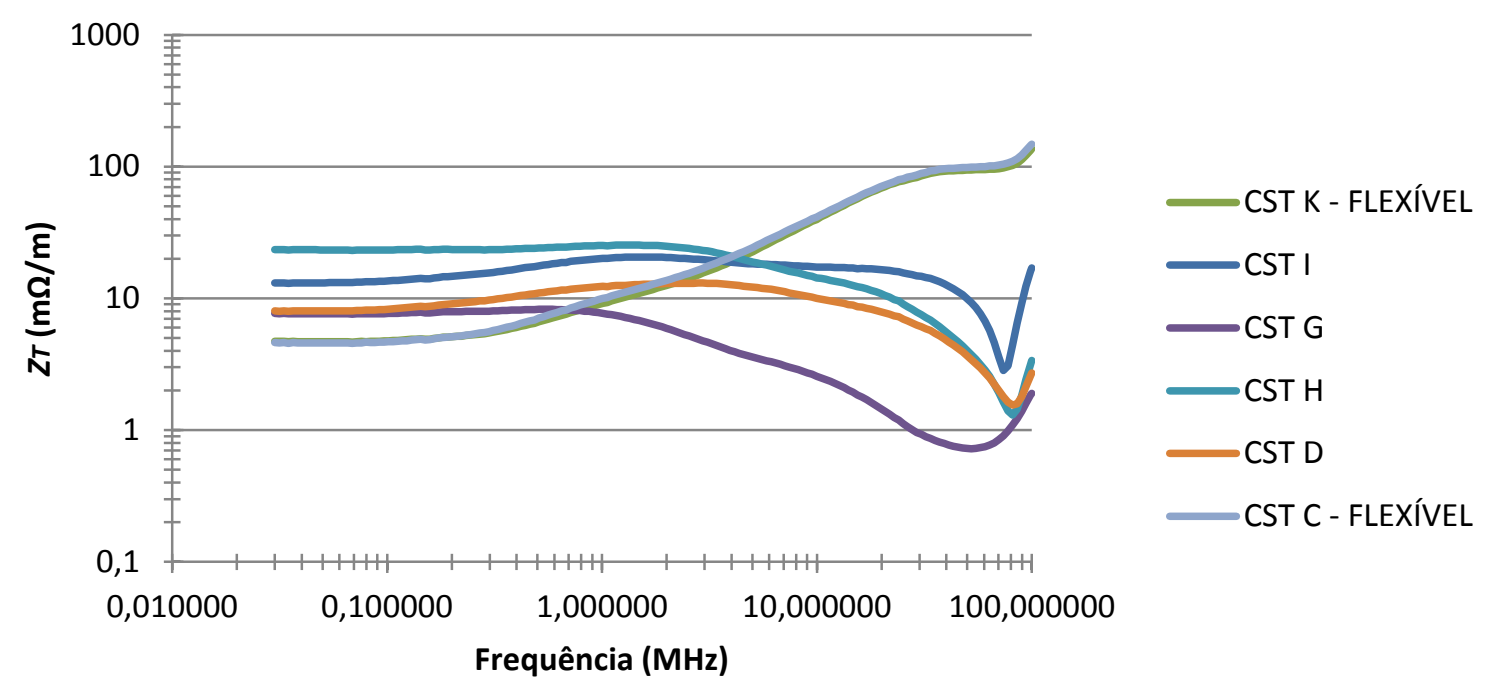

Figura 5. Comparação entre a $Z_{T}$ no domínio da frequência entre o RG213/U e o RGC213.

\section{CONCLUSÃO}

Neste trabalho, mostramos que o método triaxial pode ser utilizado para medir a impedância de transferência de cabos coaxiais e avaliar a qualidade da blindagem desses cabos. Medições realizadas com dois cabos RG123/U de fabricantes diferentes mostram similaridade entre os resultados e concordância com modelos teóricos encontrados na literatura [10]. Já as medições obtidas para os cabos RGC213 mostram uma diferença significativa entre os valores da impedância de transferência dos cabos para os diferentes fabricantes. Principalmente em altas frequências devido à diferença entre a frequência de ressonância de cada um desses cabos. Nossos resultados reforçam a necessidade de se caracterizar a impedância de transferência de cabos coaxiais e tornar esses resultados disponíveis aos usuários.

Como atividades futuras, investigaremos melhorias no aparato experimental utilizado para se obter uma melhor repetibilidade nos resultados.

AEA - Associação Brasileira de Engenharia Automotiva

Rua Salvador Correia, 80 - Aclimação

São Paulo - SP - 04109-070

Tel.: (011) 5908-4040

E-Mail: eventos@aea.org.br

\section{REFERÊNCIAS}

[1] Santos, K. M. G., Colin, A., Ramdani, M., Paulino, J. O. S. et al., "Measure of the shielding effectiveness in coaxial cables". Amsterdam. V. 1, p. 540-548, IOS Press: ISSN: 1383-728, 2010. 
[2] IEC 62153-4-3/Ed. 2.0, "Metallic communication cable test methods Part 4-3: Electromagnetic Compatibility (EMC) - Surface transfer impedance - Triaxial method", Out., 2013.

[3] IEC/TR 62153-4-1/Ed. 2.0, "Metallic communication cable test methods Part 4-1: Electromagnetic Compatibility (EMC) - Introduction to electromagnetic (EMC) screening measurements", Maio, 2010.

[4] EN 50289-1-6, "Communication cables - Specification for test methods Part 1-6: Electrical test methods - Electromagnetic performance", Set., 1999.

[5] Halme, L. e Mund, B. "EMC of Cables, Connectors and Components with Test set-up". 62nd IWCS Cable Connectivity Symposium, pp. 83-90, Nov., 2013.

[6] Démoulin, B. e Koné, L. "Shielded Cables Transfer Impedance Measurement", IEEEEMC Newsletter, pp. 30-37, Jan., 1998.

[7] Hofmeister, C., Kreisch, K., Obholz, M., Anvari, A.A.T. et. al., "Experimental Investigation of Shielding Effectiveness of Automotive HV Cables after Mechanical Stress", Proc. Of the 2013 International Symposium on Electromagnetic Compatibility (EMC Europe 2013), Brugge, Belgium, Set., 2013.

[8] Hohloch, J., Tenbohlen, S., Kohler, W., Aidam, M. et.al., "Measurement of Transfer Impedance of Components for Automotive high-Voltage power Networks", 2012 International Symposium on Electromagnetic Compatibility - EMC EUROPE, Set., 2012.

[9] Andrade, C. L., Silva, V. L., Novo, M. S. e Santos, K. M. G., "Resistor de Damping na determinação da impedância de transferência de cabos coaxiais", Artigo submetido ao Congresso SAE Brasil 2015.

[10] Leo, R. D., Cerri, G., Mariani, V. e Botticelli, R., "A Simple But Effective Way for Cable Shielding Measurement", 1999 IEEE Transactions on Electromagnetic Compatibility, Vol. 41, no 3, Ago., 1999. 